\title{
EECDC-MAC: An Energy Efficient Cooperative Duty Cycle MAC Protocol
}

\author{
Lakshmikanth Guntupalli ${ }^{1}$, Frank Y. $\mathrm{Li}^{1}$ and Xiaohu Ge ${ }^{2}$ \\ ${ }^{1}$ Dept. of Information and Communication Technology, University of Agder (UiA), N-4898 Grimstad, Norway \\ ${ }^{2}$ Dept. of Electronics and Information Engineering, Huazhong University of Science and Technology (HUST), Wuhan, China \\ ${ }^{1}\left\{\right.$ lakshmikanth.guntupalli, frank.li\}@uia.no, ${ }^{2}$ xhge@mail.hust.edu.cn
}

\begin{abstract}
In this paper, we propose a novel energy efficient cooperative duty cycle MAC (EECDC-MAC) protocol in which sensor nodes use fixed wakeup rendezvous scheduling to exchange messages and a cooperative transmission mechanism to avoid overuse of nodes with lower residual energy. Numerical results demonstrate that the EECDC-MAC protocol can prolong the entire network longevity efficiently in comparison with an existing cooperative duty cycle MAC protocol, CDC-MAC, and another popular duty cycle MAC protocol, prediction wakeup MAC (PW-MAC) protocol.
\end{abstract}

\section{INTRODUCTION}

Recently, cooperative communication has been used to balance energy consumption in wireless sensor networks (WSNs) in order to prolong network lifetime. Maximizing network lifetime by reducing battery energy consumption is a crucial design principle due to limited battery capacity of small-size nodes in WSNs. In the literature, many protocols have been proposed using duty cycle medium access control (MAC) [1-2] to extend the lifetime of WSNs, in which sensor nodes turn their radio on and off periodically to reduce energy consumption. However, a common disadvantage of these pointto-point MAC protocols is their inability to avoid energy hole.

In multi-hop WSNs, an energy hole is formed when a node around the sink is exhausted due to its heavy burden than the others. These heavily burdened nodes consume energy at a higher rate and deplete early since the data collected from the sensors is usually gathered at the sink. For example, in the network as shown in Fig. 1, a number of sending nodes, like C, D, E etc., will transmit packets to Node A via Node B. Since B needs to help other sensors to forward packets, it will consume more energy. When the energy at Node B is depleted, an energy hole is formed. To avoid this situation, one solution is to perform cooperative transmission (CT). In CT, a node can send data over longer distance across the heavily burdened node and reach the two-hop away node directly. In the literature, CDC-MAC protocol [3] is the first protocol which combines CT with duty cycling to reduce the energy hole. But that protocol spends more time on synchronization, since a sync period is always required for each packet transmission resulting in significant overhead. This drawback of CDC-MAC indeed motivated us to propose energy efficient CDC-MAC for periodic traffic.

In this paper, we propose a synchronous duty cycling MAC protocol which mitigates the energy hole by using CT from a traffic flow perspective. The proposed EECDC-MAC protocol is energy efficient because it avoids the use of sync period for every packet transmission by providing a fixed schedule in the beginning of the network initialization and consequently prolongs network lifetime. The performance of EECDC-MAC is evaluated and compared with CDC-MAC and PW-MAC. As a continuation of our earlier work, we use the same network model as the one used in [3] to evaluate the performance. Numerical results demonstrate that the proposed protocol substantially outperforms the other two studied protocols.

\section{EECDC-MAC DESIGN}

In this section, we describe the basic principle of EECDCMAC. The proposed protocol aims to balance the energy consumption of distributed nodes from the entire network point of view by exploiting the cooperative diversity gain.

Cooperative transmission works only when there are multiple active neighboring senders. Hence the basic idea of EECDC-MAC is to schedule all nodes to wake up at the same time and network synchronization is implemented by sync (S) messages in the first cycle in the network initialization phase in order to establish rendezvous for data exchange among them. From the second cycle onwards, nodes follow the schedule provided by $\mathrm{S}$ to wake up at specific fixed time instants. In CDC-MAC, synchronization is done in the sync period prior to packet transmission in the data period, whereas in EECDC$M A C$ it is a part of the data period and is done only in one cycle. The $T_{\text {cycle }}$ of a node in both CDC-MAC and EECDCMAC is shown and compared with each other in Fig. 2. Similar to CDC-MAC, data transmission in the proposed protocol is done based on the residual energy information provided by the

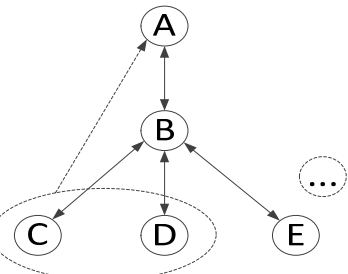

Figure 1. Network model [3] where C and D transmit to A via B in two hops, or hop over B to reach A directly by CT if the energy level at B is too low.

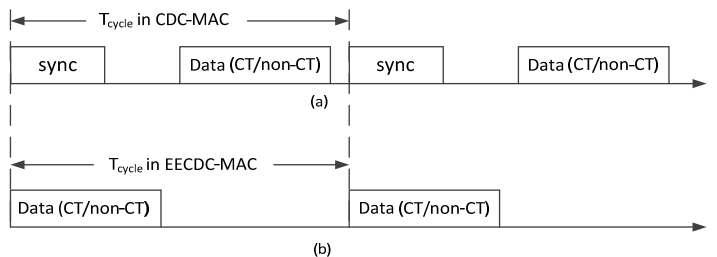

Figure 2. Duty cycle of a node (a) in CDC-MAC (b) in EECDC-MAC. 
receiving node. More specifically, if the remaining energy of the receiving node $E_{r r}$ is greater than sending node's remaining energy $E_{r s}$, i.e., $E_{r r}>E_{r s}$, then non-CT will be performed; otherwise, i.e., if $E_{r r} \leq E_{r s}$, CT will be used.

Since it is assumed that all nodes have equal energy initially, CT is initiated in the first cycle and from the second cycle onwards it is done based on the residual energy information provided by the receiver node as shown in Fig. 3 . In brief, EECDC-MAC works in a step-by-step manner as described below.

- To initiate transmission, receiver B and other sending nodes wake up at the same time. Receiver B initiates the communication by sending a sync (S) message to potential senders as a beacon. Other senders, e. g., C and D in Fig. 1, receive $\mathrm{S}$ and compete for channel access to transmit the DATA and decide whether CT should be enabled or not.

- To perform CT, the node which has got channel access broadcasts a call for cooperation (CFC) message along with DATA to initiate CT. In Fig. 3(a), the CFC is broadcast by Node $\mathrm{C}$ which is a sending node to recruit other nodes as cooperators to initiate CT. Upon receiving the CFC packet, the receiving node $\mathrm{B}$ will go to sleep. After a SIFS, CT by Node $\mathrm{C}$ and the selected cooperators will be concurrently performed [4]. After receiving the DATA packet, Node A will respond with ACK to Node $B$ and then $B$ forwards it to $\mathrm{C}$ and $\mathrm{D}$. In the current cycle, Node $\mathrm{B}$ has to wake up again before the arrival of the ACK packet since ACK for CT will be sent back to Node $\mathrm{C}$ via Node B. In order to capture ACK sent from A, Node B needs to wake up at the instant (2 DATA +2 SIFS) seconds after it goes to sleep. Although it consumes energy to reactivate Node B, we can still conserve energy instead of keeping Node B always awake.

- From the second cycle onwards, the receiving node sends a ready to receive (RTR) message that contains residual energy and distance information together with the request for data transmission to sending nodes. Then the sending node has to decide whether to do CT or not [5] by comparing with its own residual energy with the residual energy of the receiving node. To perform CT after the first cycle, nodes follow the same procedure as explained above. In general, the nodes with their remaining energy above Node B's become cooperator candidates. They will store the overheard DATA message and then perform CT after the SIFS interval. If CT fails cooperators, retransmission of CT (reCT) will be initiated.

- To perform non-CT, after receiving an RTR message, both sending nodes wait for a SIFS period and then contend for channel access using a random backoff scheme. In Fig. 3(b), it is assumed that Node C got channel access and Node D lost the competition (indicated by dashed line packet). Now, Node C compares its residual energy with the receiver's remaining energy and if its residual energy is lower than B's then it will send DATA to receiver B. Receiver B sends an acknowledgement (ACK) for the received DATA. At the same time, other nodes which lost the previous contention will freeze their backoff counters. They resume counting to send DATA when the medium is sensed as free again.
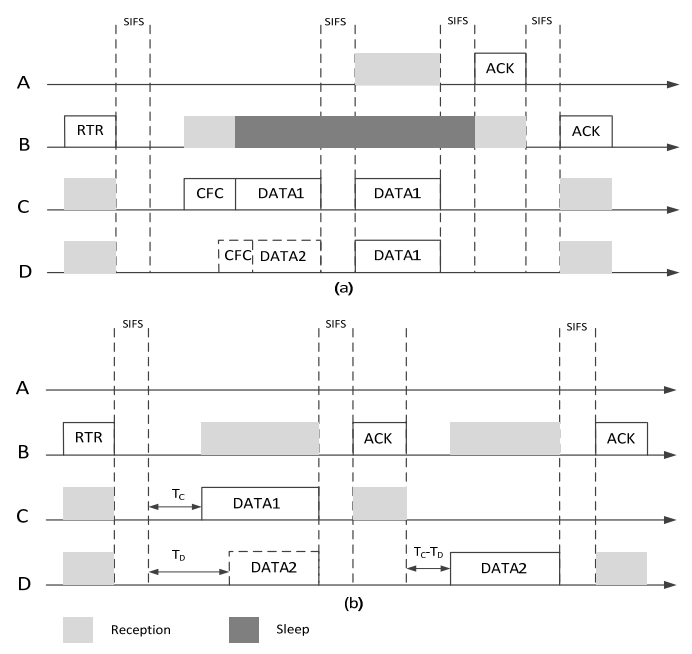

Figure 3. (a) CT (b) non-CT in EECDC-MAC.

\section{Performance Evaluation}

This section gives an idea how to analyze the energy performance of EECDC-MAC and presents the numerical results in comparison with CDC-MAC and PW-MAC. The numerical results are evaluated using MATLAB and are based on the following analysis which is for one cycle. The analysis is based on the network model described in Section I where each node generates packets at a fixed interval $T_{\text {cycle }}$ for an environmental monitoring application [1]. Consider five radio states: transmitting, receiving, listening, idle and sleeping, each drawing the power of $P_{t x}, P_{r x}, P_{\text {listen }}, P_{\text {idle }}$ and $P_{\text {sleep }}$ respectively, and that they spend in each state for $T_{t x}, T_{r x}, T_{\text {listen }}, T_{\text {idle }}$ and $T_{\text {sleep }}$ period of time respectively. The time with radio being asleep is simply sleep time and it is given by

$$
T_{\text {sleep }}=T_{\text {cycle }}-T_{t x}-T_{r x}-T_{\text {listen }}-T_{\text {idle. }} \text {. }
$$

The energy consumption, per node, is given by:

$$
\begin{aligned}
E & =E_{c s}+E_{t x}+E_{r x}+E_{\text {idle }}+E_{\text {sleep }} \\
& =P_{\text {listen }} T_{c s}+P_{t x} T_{t x}+P_{r x} T_{r x}+P_{\text {idle }} T_{\text {idle }}+P_{\text {sleep }} T_{\text {sleep } .} .
\end{aligned}
$$

The networking parameters used in the analysis are based on the Mica2 radio (Chipcon CC1000 [1]). Then the energy consumption by both receiver and $\mathrm{CT}$ initiators can be calculated based on their operation as illustrated in Fig. 3. To evaluate the performance of CDC-MAC, an extra sync period is included in each cycle, and PW-MAC is evaluated using only non-CT transmission.

\section{A. Lifetime Comparison of Different Protocols}

Fig. 4 depicts the lifetime comparison of these three protocols with packet size $L_{\text {data }}=50$ bytes, $N_{c}=2, T_{\text {cycle }}=1 \mathrm{sec}$ where the initial energy contained by each node is $1 \mathrm{~J}$. From the zoomed-in part in Fig. 4, it can be observed that the residual energy of PW-MAC decreases linearly whereas the residual energy of EECDC-MAC and CDC-MAC protocols is decreasing alternatively between the $\mathrm{CT}$ and the non-CT periods as time goes. It shows that both EECDC-MAC and CDC-MAC initiate CT when necessary. However, from the 


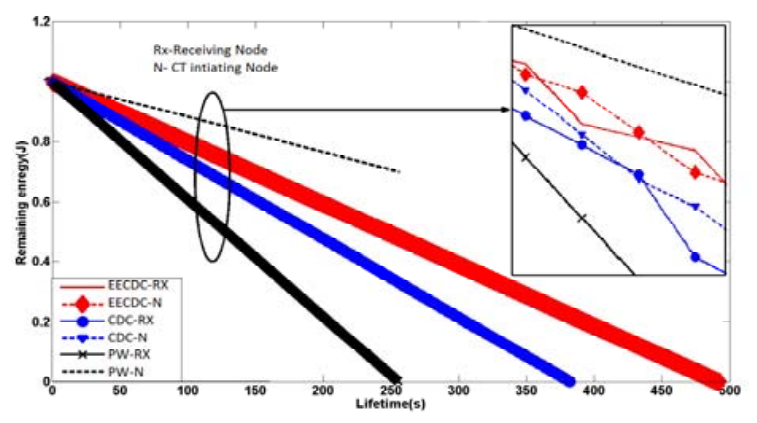

Figure 4. Lifetime comparison of the three protocols.

network lifetime point of view (the first node depletes in the network), EECDC-MAC has achieved maximum lifetime. The lifetime of EECDC-MAC is $28.70 \%$ longer than CDC-MAC's and $92.18 \%$ longer than PW-MAC's. The reason is as follows. EECDC-MAC avoids the use of extra sync period in each cycle, so, the energy consumption rate is lower when compared with CDC-MAC. In the case of PW-MAC, since only regular data packet transmission (non-CT) is used, the energy consumption rate is even higher than the CDC-MAC case. Hence the proposed EECDC-MAC has longest lifetime.

\section{B. Balanced Network Lifetime}

EECDC-MAC shows its superiority in balancing network lifetime as well. To illustrate that, we compare the proposed protocol with the other studied protocols from the balanced network lifetime point of view by redefining network lifetime as the time that the last node drains its energy. In Fig. 4, we find that Node B which is represented by RX depletes much earlier than the other nodes when PW-MAC is used. It is further shown in Fig. 4 that, even though Node $\mathrm{C}$ which is represented by $\mathrm{N}$, still has lot of residual energy, it is disconnected from the network due to the earlier depletion of $\mathrm{RX}$. This is because that PW-MAC does not use CT that saves energy of the node prone to energy hole. Node B is overused in PW-MAC even though it has pretty low residual energy. In EECDC-MAC and CDC-MAC, however, it is found that almost all nodes run out of energy at the same time. Furthermore, EECDC-MAC exhibits an advantage on network lifetime over CDC-MAC and PW-MAC as shown in Fig. 4.

\section{Effect of Packet Size and Number of Cooperating Nodes}

For the results in Fig. 5, the packet size is varied from 50 bytes to 250 bytes and the number of cooperators $N_{c}$ is fixed to 2. It is found that the lifetime of all protocols decreases as the data packet size increases since more energy is needed to transmit the larger size packet. Assume that all the cooperators are in the range of one hop away from the receiving node and the distance between the receiving node and a cooperating node is the same for all nodes. The lifetime variation when $N_{c}$ varies from 2 to 5 is shown in Fig. 6, while the packet size is fixed to 50 bytes. The lifetime of all protocols is decreased when more cooperator nodes are involved. In the non-CT period, a cooperating node will be a competing node. So, heavy burden will be introduced on the receiving node since it has to serve all the competitors. Correspondingly, the receiver has to spend

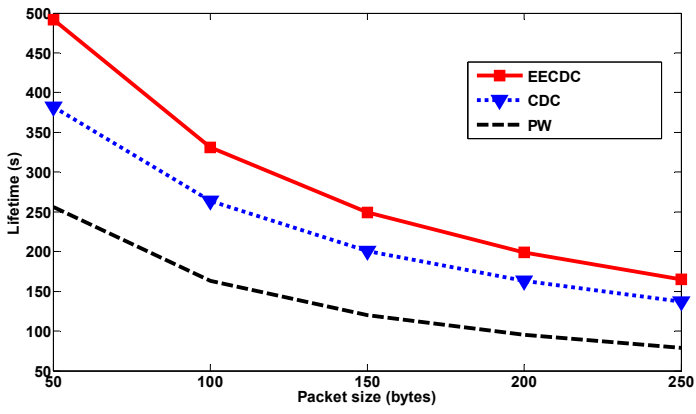

Figure 5. Lifetime variation with packet size.

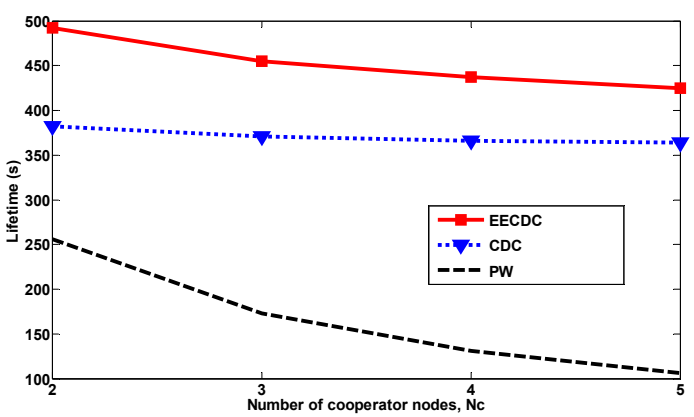

Figure 6. Lifetime variation with $\mathrm{N}_{\mathrm{c}}$.

more energy in the non-CT period when the number of nodes is larger and hence the lifetime is reduced. Again, EECDC-MAC has longer lifetime in both cases.

\section{CONCLUSIONS}

In this paper, we have proposed EECDC-MAC, an energy efficient cooperative duty cycle MAC protocol for periodic traffic generating applications. EECDC-MAC mitigates the effect of energy hole in WSNs by performing CT when necessary based on the residual energy information of both senders and receivers. EECDC-MAC avoids the use of synchronization for each packet transmission to extend the network lifetime of WSNs. The results demonstrate that the EECDC-MAC achieves longer lifetime in studied cases.

\section{REFERENCES}

[1] W. Ye, F. Silva, and J. Heidemann, "Ultra-low duty cycle MAC with scheduled channel polling," in Proc. of ACM SenSys, pp. 321-333. Boulder, Colorado, USA, Nov. 2006.

[2] L. Tang, Y. Sun, O. Gurewitz and D. B. Johnson, "PW-MAC: An energy efficient prediction-wakeup MAC protocol for wireless sensor networks", in Proc. of INFOCOM, Shanghai, China , April 2011.

[3] H. Jiao, M. A. Ingram and F.Y. Li, "A cooperative lifetime extension MAC protocol in duty cycle enabled wireless sensor networks," in Proc. of IEEE MILCOM , Baltimore, MD, USA, 7-10 Nov. 2011.

[4] H. Jung, Y. J. Chang, and M. A. Ingram, "Experimental range extension of concurrent cooperative transmission in indoor environments at 2.4 GHz," in Proc. of IEEE MILCOM, San Jose, CA, USA, Nov. 2010.

[5] J. W. Jung and M. A. Ingram, "Residual-Energy-Activated Cooperative Transmission (REACT) to avoid the energy hole," in Proc. of IEEE ICC Workshop on Cooperative and Cognitive Mobile Networks (CoCoNet3), Cape Town, South Africa, May 2010. 